\title{
Aberrant FGF signaling promotes granule neuron precursor expansion in SHH subgroup infantile medulloblastoma
}

\author{
Odessa R. Yabut ${ }^{1, *}$, Hector Gomez ${ }^{1}$, Jessica Arela1, Jesse Garcia Castillo, Thomas Ngo1, 2, and \\ Samuel J. Pleasure ${ }^{1,3, *}$ \\ 1Department of Neurology, Weill Institute for Neuroscience, University of California San Francisco, San Francisco, California \\ ${ }^{2}$ Department of Psychiatry, Weill Institute for Neuroscience, University of California San Francisco, San Francisco, California, USA. \\ ${ }^{3}$ Programs in Neuroscience and Developmental Biology, Eli and Edythe Broad Center of Regeneration Medicine and Stem Cell \\ Research, University of California San Francisco, California, USA \\ *To whom correspondence should be addressed: e-mail: samuel.pleasure@ucsf.edu (S.J.P.) or odessa.yabut@ucsf.edu (O.R.Y.)
}

\begin{abstract}
Mutations in Sonic Hedgehog (SHH) signaling pathway genes, e.g. Suppressor of Fused (SUFU), drive granule neuron precursors (GNP) to form medulloblastomas (MB $\mathrm{MHH}$ ). However, how different molecular lesions in the Shh pathway drive transformation is frequently unclear, and in particular, SUFU mutations in the cerebellum seem distinct. In this study, we show that fibroblast growth factor 5 (FGF5) signaling is integral for many infantile MBSHH cases. We found that FGF5 expression is uniquely upregulated in infantile $\mathrm{MB}^{\mathrm{SHH}}$ tumors. Also, in mice carrying loss-of-function SUFU mutations (Sufu-cKO), FGF5 is ectopically expressed specifically along the secondary fissure where GNPs harboring preneoplastic DNA lesions are massively expanded and FGFR signaling is also ectopically activated in this region. Treatment with an FGFR antagonist rescues the severe GNP hyperplasia and restores cerebellar architecture. Thus, direct inhibition of FGF signaling may be a promising and novel therapeutic candidate for infantile $\mathrm{MB}^{\mathrm{SHH}}$.
\end{abstract}

\section{Introduction}

Medulloblastoma (MB) is the most common malignant brain tumor in children, with half of cases diagnosed before the age of 5 (Ostrom et al., 2016; Ward et al., 2014). MB is classified into four distinct subgroups: wingless (Wnt; Wnt subgroup), sonic hedgehog ( $\mathrm{SHH}$; $\left.\mathrm{MB}^{\mathrm{SH}}\right)$, group 3, and group 4 (Kool et al., 2012). MB ${ }^{\mathrm{SHH}}$ accounts for $\sim 30 \%$ of all MB patients and is generally thought to be due to activating or inactivating mutations in genes along the SHH signaling pathway. However, there is considerable patient-to-patient heterogeneity between $\mathrm{MB}^{\mathrm{SHH}}$ patients including variations in age of onset, molecular signatures, tumor progression, treatment response, and relapse (Cavalli et al., 2017; Schwalbe et al., 2017). In particular, infantile MB is especially heavily represented in the $\mathrm{MB}^{\mathrm{SHH}}$ subgroup and these patients have worse prognosis for many reasons (Remke and Ramaswamy, 2018). We believe that distinctions between particular mutant genes in the $\mathrm{MB}^{\mathrm{SHH}}$ subgroup may predict tumor progression and treatment response. This presents an opportunity to develop highly effective targeted therapies by understanding of the normal development of the cerebellar granule precursors (the cell of origin for MB) as well as factors affecting tumor initiation and maintenance unique to distinct $\mathrm{MB}^{\mathrm{SHH}}$ tumor subtypes.

Human MB tumors and $\mathrm{MB}^{\mathrm{SHH}}$ mouse models show distinct cerebellar anatomic origins according to initiating mutations (Tan et al., 2018; Wefers et al., 2014). In particular, a large proportion of infantile-onset $\mathrm{MB}^{\mathrm{SHH}}$ originate from the cerebellar midline (vermis). Effective and targeted anti-tumor therapies for this most deadly group of $\mathrm{MB}$ will require understanding the convergence of oncogenic mutations with spatiotemporal factors regulating regional development. Our studies focus on
Suppressor of Fused (SUFU) mutations which comprise approximately $30 \%$ of tumors in infants with $\mathrm{MB}^{\mathrm{SH} H}$ (MB ${ }^{\mathrm{SHH}}-$ SUFU). Importantly, MB ${ }^{\text {SHH-SUFU }}$ patients have worse prognosis and higher rates of local recurrence than other $\mathrm{MB}^{\mathrm{SHH}}$ subtypes (Guerrini-Rousseau et al., 2018; Kool et al., 2014; Schwalbe et al., 2017). In addition, available SHH-targeted treatments for $\mathrm{MB}^{\mathrm{SHH}}$ act specifically on proteins upstream of SUFU and are therefore ineffective for MB ${ }^{\text {SHH-SUFU }}$ patients (Kool et al., 2014). Intriguingly, tumors associated with SUFU mutations exclusively originate from the cerebellar midline (vermis) (Tan et al., 2018). The poor prognosis, early occurrence, anatomic distribution and lack of targeted therapy for MB ${ }^{\text {SHH-SuFu }}$ patients make detailed understanding of the drivers of oncogenesis in this group of great importance.

Sufu acts as an intracellular modulator of SHH signaling. Briefly, the SHH signaling pathway is initiated after binding of extracellular SHH ligands to the transmembrane receptor Patched 1 (Ptch1). This relieves Ptch1 inhibition of the transmembrane protein, Smoothened (Smo), and enables the initiation of a cascade of intracellular events promoting the activator function of the transcription factors, Gli1, Gli2, or Gli3. Sufu modulates SHH signaling by ensuring the stability of Gli transcription factors or by promoting the formation of the repressor forms of Gli2 (Gli2R) or Gli3 (Gli3R). Thus, depending on developmental context, loss of Sufu can lead to activation or repression of SHH responsive signaling. In the developing cerebellum, Sufu dysfunction is associated with abnormal development of granule neuron precursors (GNP), the cells of origin for $\mathrm{MB}^{\mathrm{SHH}}$ (Jiwani et al., 2020; Kim et al., 2011, 2018; Kong et al., 2019; Vanner et al., 2014; Vladoiu et al., 2019; Yin et al., 2019). GNPs populate the external granule layer (EGL) along the cerebellar 
A
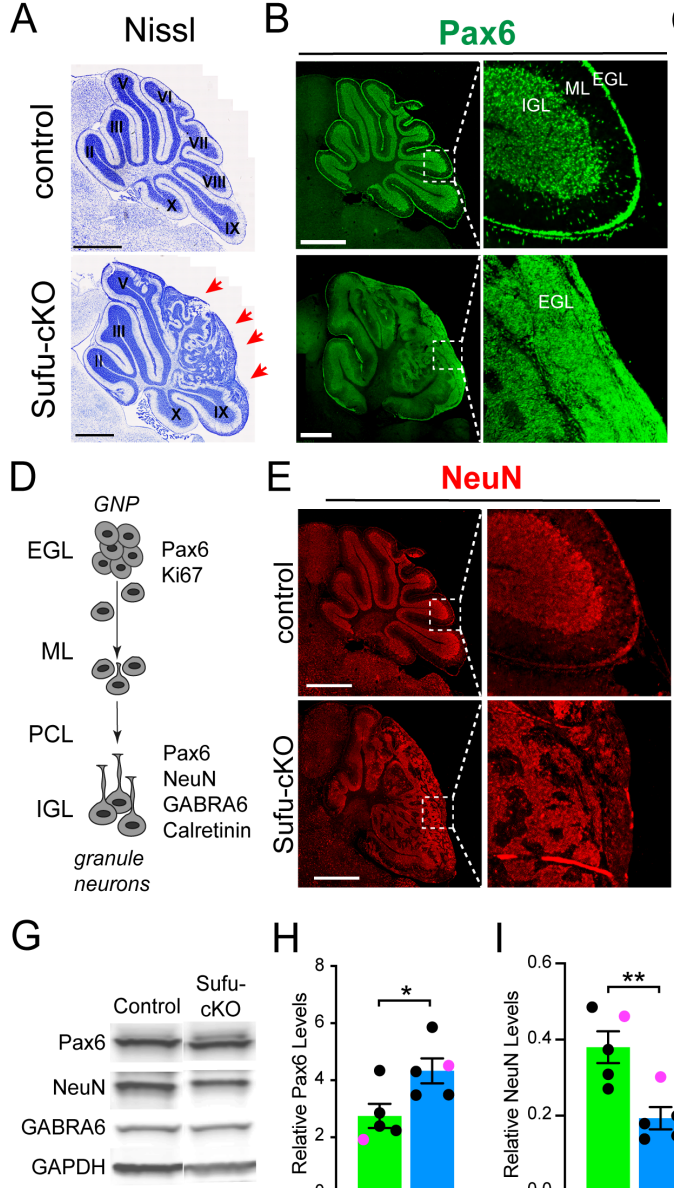

C Pax6 Ki67
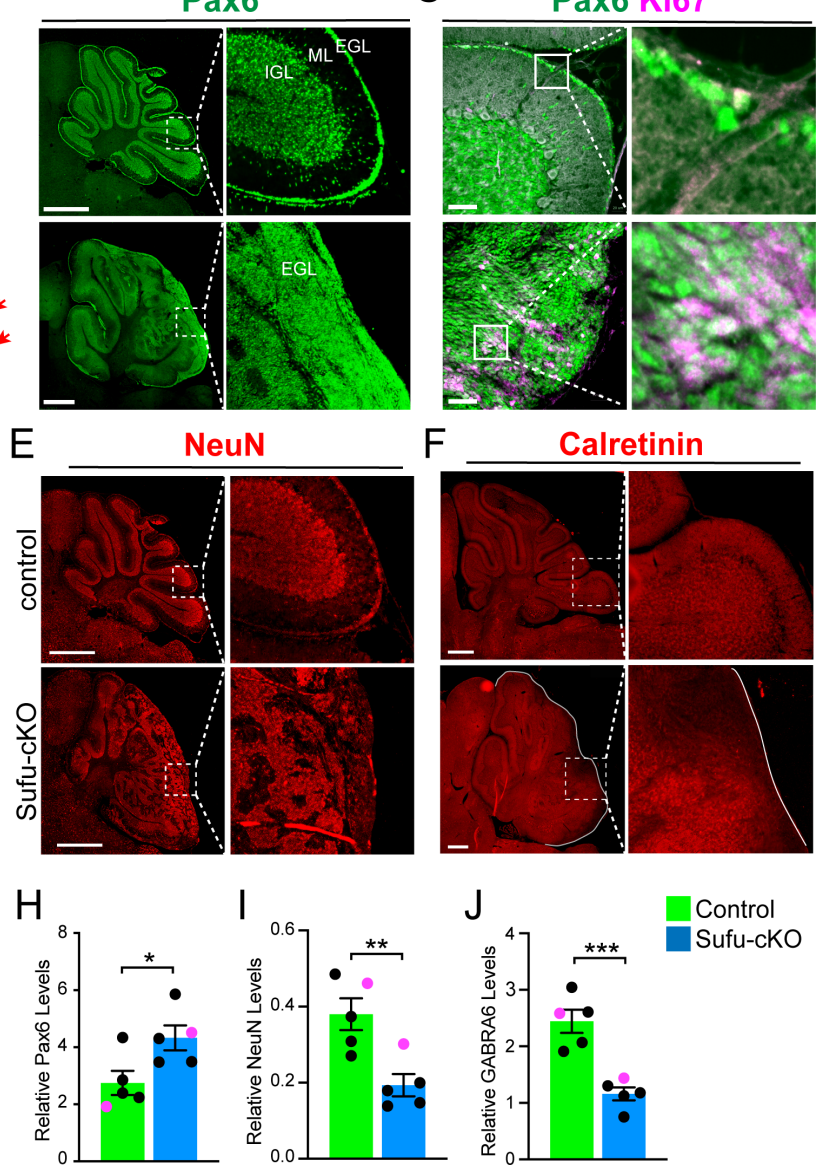

Figure 1: Cerebellar granule neuron precursors fail to differentiate and continue to proliferate in Sufu-cKO mice. (A) Nissl staining of P14 Sufu-cKO and control cerebelli. Red arrows point to severe hyperplasia between lobules $V$ and IX. (B) Immunofluorescence staining with the granule neuron lineage marker Pax6 in P14 SufucKO and control cerebelli. Boxed regions show Pax6-expressing cells in areas with severe hyperplasia between lobules $\mathrm{V}$ and IX resulting in the expansion of external granule layer (EGL) and indistinguishable molecular layer (ML) and internal granule layer (IGL). (C) Double-immunofluorescence staining with Pax6 (green) and the proliferation marker, Ki67 (magenta) on the P14 Sufu-cKO and control cerebelli. Boxed regions magnify the EGL where proliferating Ki67-expressing cells localize. (D) Schematic diagram of the granule neuron lineage progression from the EGL where GNPs proliferate and become postmitotic prior to migrating into the $M L$ and the purkinje cell layer $(\mathrm{PCL})$ to reach the IGL where granule neurons fully differentiate and express mature granule neuron markers. (E-F) Immunofluorescence staining with markers for mature granule neurons NeuN and calretinin the P14 Sufu-cKO and control cerebelli. Boxed regions show areas of presumptive cerebellar lobule VIII in Sufu-cKO and controls where NeuN (E) and calretinin (F) signals are lower. (G-J) Western blot analysis (G) of mature granule neuron markers Pax6, NeuN and GABRA6 protein levels normalized against GAPDH show higher relative levels of Pax6 $(H)$, but lower levels of NeuN (I) and GABRA6 $(\mathrm{J})$ in whole cerebellar protein lysates obtained from the P14 Sufu-cKO mice compared to littermate controls. Individual values in magenta correspond to western blot data in $G .{ }^{*} p<0.05,{ }^{* \star} p<0.01,{ }^{\star \star *} p<0.001$. Scale bars $=1000$ $\mu \mathrm{m}(\mathrm{A}, \mathrm{B}, \mathrm{E}, \mathrm{F}) ; 50 \mu \mathrm{m}(\mathrm{C})$.

perimeter where these cells expand prior to differentiating into granule neurons. SHH signaling is a major driver of GNP proliferation and differentiation in neonatal stages. However, other mitogenic pathways can also influence granule neuron lineage progression at this stage (Leto et al., 2016). Since targeted treatments for $\mathrm{MB}^{\mathrm{SHH}}$ are ineffective for MB ${ }^{\text {SHH-SUFu }}$ patients (Kool et al., 2014), it is crucial to determine if and how SUFU loss-of-function (LOF) affects the activity of concurrent local signaling pathways influencing granule neuron development since these may be potent potential targets for anti-tumor therapy.
In this study, we show that ectopic expression of FGF5 in the neonatal cerebellum of mice lacking SUFU ( $h G F A P$-Cre;Sufuflff, hereto referred as Sufu-cKO) disrupts granule neuron lineage expression. Expansion of FGF5expressing cells activates FGF signaling in EGLlocalized cells resulting in disrupted GNP differentiation and the accumulation of GNPs. Strikingly, acute pharmacological inhibition of FGF signaling in neonatal Sufu-cKO mice results in near-complete rescue of these defects, including a restoration of cerebellar histoorganization. Furthermore, we show that FGF5 expression is increased particularly in tumors of infantile $\mathrm{MB}^{\mathrm{SHH}}$ patients. Thus, our findings identify FGF5 as a potential biomarker for a subset of patients with infantile $\mathrm{MB}^{\mathrm{SHH}}$ who may be responsive to FGFR-targeting therapies.

\section{Results}

Cerebellar granule neuron precursors fail to differentiate and continue to proliferate in SufucKO mice

To specifically examine granule neuron lineage progression in the absence of Sufu, we generated Sufu-cKO mice, in which Sufu is specifically deleted in cerebellar neural stem cells (NSC) destined to generate GNPs in the embryonic day (E)13.5 cerebellar rhombic lip (Zhuo et al., 2001). At postnatal day (P) 14, SufucKO mice exhibit a severely disorganized cerebellum. Nissl staining shows densely populated areas leading to the lack of distinguishable laminar and follial structures in the P14 Sufu-cKO cerebellum, particularly areas between lobules VI and IX where these defects are most severe (Fig. 1A, arrows). Immunofluorescence staining for Pax6, a marker for the granule neuron lineage, shows that the P14 Sufu-cKO cerebellum is densely packed with Pax6-positive (Pax6+) cells (Fig. 1B). Thus, in accordance with previous observations(Yin et al., 2019), our findings confirm that loss of Sufu in late-embryonic stage cerebellar NSCs results in the expansion of granule neuron lineage cells.

To examine whether Pax6-expressing (Pax6+) cells in the P14 Sufu-cKO cerebellum represent differentiated granule neurons or proliferating GNP, we performed immunostaining with Pax6 and the proliferation marker Ki67. We find that Ki67+ cells are present and form aggregates along the perimeter of the cerebellum (Fig. 1C). Further, many Ki67+ cells are Pax6+ suggesting expansion of GNPs (Fig. 1C, boxed region). Thus, a significant proportion of Pax6+ GNPs remain proliferative in the P14 Sufu-cKO, a time point at which granule neurons have normally differentiated and migrated through the molecular layer (ML) to reach the internal granule layer (IGL) and matured (Fig. 1D). Immunostaining for markers of differentiated granule neurons (NeuN and Calretinin) are also reduced in the P14 Sufu-cKO 
cerebellum. This is especially apparent in areas where Ki67+ cells are most enriched (Fig. 1E-F). Western blots also show lower levels of NeuN, and GABRA6 in the P14 Sufu-cKO cerebellum despite higher Pax6 protein levels relative to the P14 control cerebellum (Fig. 1G-J). Altogether, these findings establish that the majority of Pax6+ granule neuron lineage cells fail to fully differentiate and maintain an immature proliferative state in the absence of Sufu.

The PO Sufu-cKO cerebellum is expanded with increased numbers of granule neuron precursors

The reduction in differentiated granule neurons by P14 in the Sufu-cKO cerebellum indicates that there must be defects at earlier stages. Since these defects are not present when Sufu is deleted after GNPs are specified (Yin et al., 2019), we postulated that disruptions in GNP lineage progression originate prior to GNP differentiation. We therefore examined the behavior of GNPs at P0, when GNP expansion and differentiation has just begun. Indeed, at P0, there is a visible increase in measured cerebellar size and expansion of Pax6+ GNPs in the EGL of Sufu-cKO cerebellum compared to controls (Fig. 2A-B). This phenotype is due to significant expansion of the Pax6+ GNP-populated EGL regions in the P0 Sufu-cKO cerebellum (Fig. 2C). Notably, this effect is regionally specific with expansion of Pax6+ GNPs particularly along the secondary fissure (EGL Region B, arrow in Fig. 2A) compared to other EGL areas (EGL Regions $A$ and $C$ ) in the P0 Sufu-cKO cerebellum (Fig. 2D). Thus, mice lacking Sufu have an enlarged cerebellum due to excessive Pax6+ GNP proliferation at very early postnatal stages indicating A

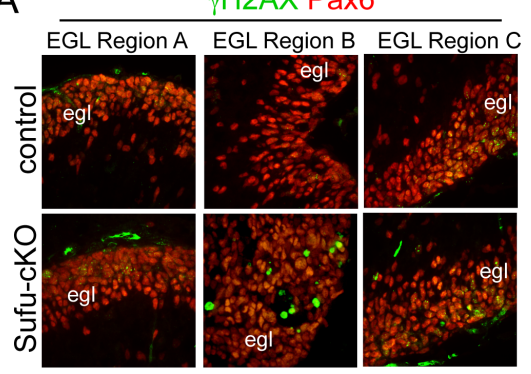

B

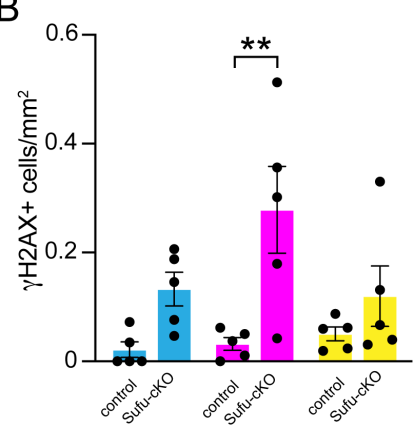

C
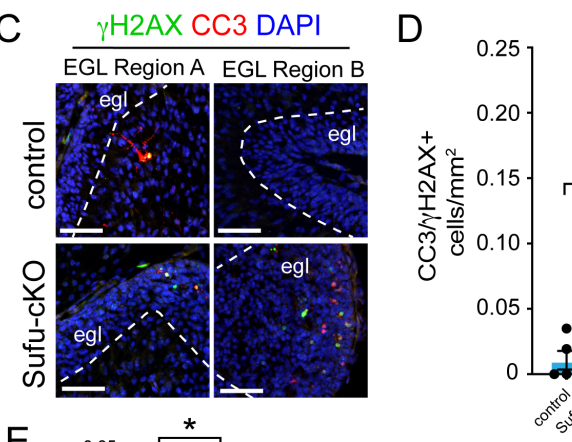

E

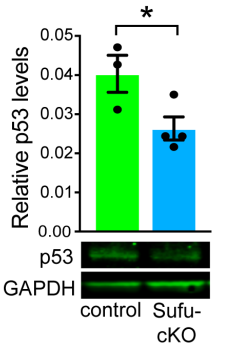

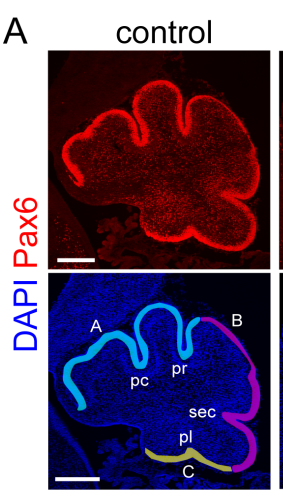
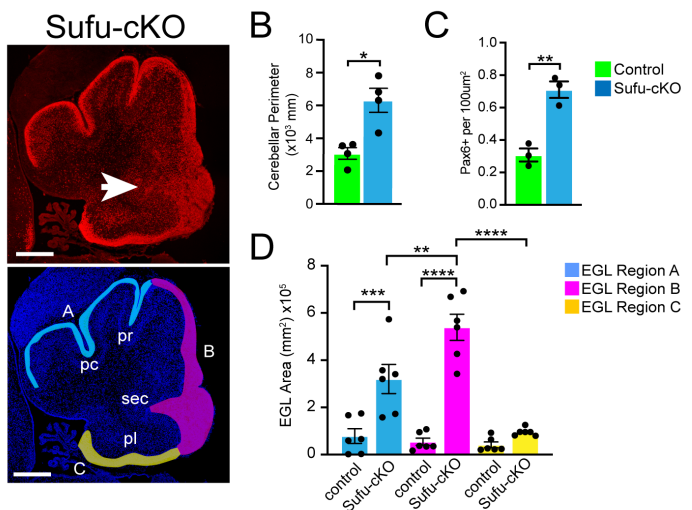

Figure 2: The newborn Sufu-cKO cerebellum is expanded with increased numbers of granule neuron precursors. (A) Pax6 (red) and DAPI (blue) immunofluorescence staining of the PO Sufu-cKO and control cerebelli. Arrow points to severely expanded EGL region B in the PO Sufu-cKO cerebellum. EGL regions are designated in DAPI-labeled sections as A (light blue), B (magenta), and $C$ (yellow). Each region encompasses specific fissures: the preculminate (pc) and primary (pr) fissures for Region A, the secondary (sec) fissure for Region B, and the posterolateral ( $\mathrm{pl}$ ) fissure for Region C. Scale bars: Scale bars $=250 \mu \mathrm{m}$. (B-D) Quantification and comparison of the cerebellar perimeter (B), total area occupied by densely packed Pax6+ cells (C), and size of specific EGL regions (D) between PO Sufu-cKO and control cerebelli. ${ }^{*} p<0.05,{ }^{* *} p<0.01$, ${ }^{\star \star *} p<0.001,{ }^{* \star \star *} p<0.0001$.

defects in controlling early steps in GNP expansion and differentiation.

Pre-neoplastic lesions are present in GNPs of the newborn Sufu-cKO cerebellum

The massive expansion of GNPs in the newborn SufucKO EGL prompted us to investigate the nature of these precursors. Particularly, we wondered if these cells are vulnerable to oncogenic insults like replication stressinduced DNA double-strand breaks (DSBs), which severely compromise genomic stability and can trigger MB oncogenesis as previously observed (Frappart et al., 2009; Pazzaglia et al., 2006). To detect DSBs, we performed immunostaining for phosphorylated H2AX ( $\gamma \mathrm{H} 2 \mathrm{AX})$, which is produced as an early cellular response to DSBs (Mah et al., $2010)$. We found many $(\gamma \mathrm{H} 2 \mathrm{AX}$-positive $((\gamma \mathrm{H} 2 \mathrm{AX}+)$ cells in the EGL of PO Sufu-cKO cerebellum while these cells are essentially absent in controls (Fig. 3A). $(\gamma \mathrm{H} 2 \mathrm{AX}+$ cells also co-label with Pax6 indicating that DSBs are occurring in granule neuron lineages in the Sufu-cKO cerebellum - the origin cells for MB. The numbers of $(\gamma \mathrm{H} 2 \mathrm{AX}+$ cells across all EGL regions of the P0 Sufu-cKO cerebellum are elevated, with a

Figure 3: Evidence of pre-neoplastic lesions and high rates of cell death in Sufu-cKO granule neuron precursors. (A) Double-immunofluorescence staining with Pax6 (red) and $(\gamma \mathrm{H} 2 \mathrm{AX}$ (green), a marker for double-strand DNA breaks in specific EGL regions of the PO Sufu-cKO and control cerebelli. (B) Quantification of $(\gamma \mathrm{H} 2 \mathrm{AX}+$ cells in each cerebellar region of PO control and Sufu-cKO mice. ${ }^{\star *} p<0.01$. (C) Doubleimmunofluorescence staining with $(\gamma \mathrm{H} 2 \mathrm{AX}$ (green) and cleavedcaspase 3 (CC3; red), a marker for apoptotic cells, and DAPI labeling in regions $A$ and $B$. Scale bars $=50 \mu \mathrm{m}$ (D) Quantification of the density of cells labeled with $\mathrm{CC} 3$ and $\left(\gamma \mathrm{H} 2 \mathrm{AX}\right.$ within each EGL regions. ${ }^{*} p<0.05,{ }^{* * *} p<0.001$. (E) Western blot analysis of p53 protein levels in P0 control and Sufu-cKO cerebellar protein lysates. ${ }^{*} p<0.05$. (F) Hematoxylin and Eosin (H\&E) staining of P60 control, Sufu-cKO, Sufu;p53dKO cerebelli. Scale bars $=500 \mu \mathrm{m}$ 
bioRxiv preprint doi: https://doi.org/10.1101/2021.06.23.449636; this version posted June 24,2021 . The copyright holder for this preprint (which was not certified by peer review) is the author/funder, who has granted bioRxiv a license to display the preprint in perpetuity. It is made available under aCC-BY-NC-ND 4.0 International license.

\section{Aberrant FGF signaling in SHH subgroup infantile medulloblastoma}

A

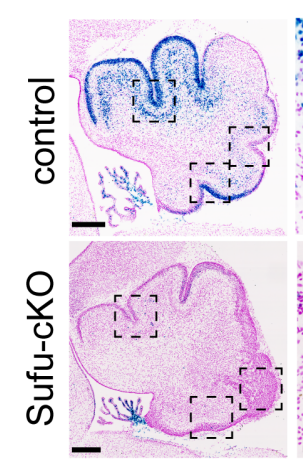

$\mathrm{B}$ Whole cerebellum

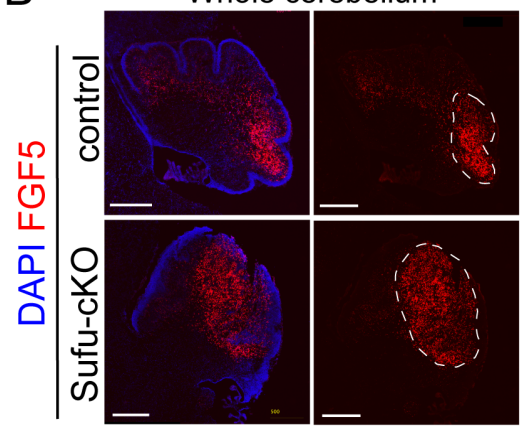

C Gli1-LacZ Fast Red EGL Region A EGL Region B EGL Region C

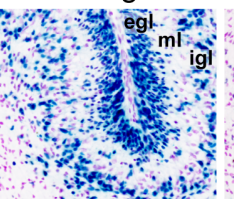
igl $\mathrm{ml}$ - $\quad \mathrm{ml}^{\mathrm{ml}}$ $\mathrm{ml}$ eglo

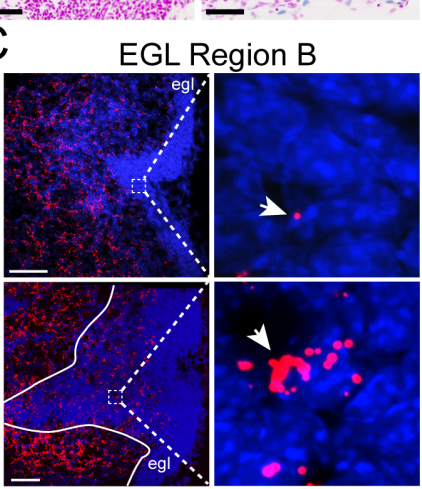

Figure 4: Downregulated Sonic Hedgehog signaling activity and increased expression of FGF5 ligands in the PO Sufu-cKO cerebellum. (A) Visualization of SHH signaling activity via b-galactosidase activity (blue) in the PO Sufu-cKO and control mice carrying the Gli1-LacZ transgene. Boxed areas represent enlarged $E G L$ regions $A, B$, and $C$ showing the distribution of Gli1LacZ+ cells across cerebellar layers. egl, external granule layer; $\mathrm{ml}$, molecular layer; igl, internal granule layer. Scale bars $=200 \mu \mathrm{m}$ and $50 \mu \mathrm{m}$ (enlarged regions). (B) Fluorescent in situ hybridization using RNAScope probes against FGF5 mRNA (red) and DAPI labeling in the PO Sufu-cKO and control whole cerebellum. Areas with high levels of FGF5 expression are outlined. Scale bars $=500 \mu \mathrm{m}$. (C) FGF5, as detected by fluorescent in situ hybridization, is ectopically expressed by cells in the EGL and adjacent regions of Region $B$. Boxed areas within the EGL show DAPI-labeled cells expressing visibly high levels of FGF5 (arrowheads) in the EGL of PO Sufu-cKO cerebellum compared to controls. Scale bars $=50 \mu \mathrm{m}$.

significant increase observed in Region B compared to controls (Fig. 3B). Nevertheless, tumors do not readily form in the Sufu-cKO cerebellum (data not shown), aligning with previous observations (Yin et al., 2019), indicating either timely repair of DSBs or that apoptosis is triggered in GNPs with significant genomic instability. Indeed, double-labeling with cleaved Caspase 3 (CC3) and $\gamma \mathrm{H} 2 \mathrm{AX}$ show a significantly higher number of double-labeled cells in EGL Regions A and B (Fig. 3C-3D). Among the downstream targets of DSB repair pathways is p53, which when activated mediates cell death to suppress tumor formation (Gao et al., 2000). In the P0 Sufu-cKO cerebellum, p53 protein is present, albeit significantly reduced (Fig. 3E). This level of p53 reduction may be driving an increase in DSBs yet still sufficient to induce apoptotic pathways. Supporting this, complete ablation of both p53 and Sufu in GNPs (Sufu;p53-dKO) results in massive tumors forming in the cerebellum within 2 months (Fig. 3F). These findings suggest that in the absence of Sufu, the failure of GNPs to transition into fully differentiated granule neurons compromises genomic stability and renders GNPs extremely vulnerable to tumor formation with a second molecular hit.
Region-specific expansion of GNPs in the PO Sufu-cKO cerebellum coincides with increased FGF5 expression

GNP proliferation is well known to be controlled by SHH signaling activity (Wechsler-Reya and Scott, 1999). However, recent studies have shown that Sufu loss of function (LOF) in the developing cerebellum has complicated effects on the activity of Gli transcription factors at neonatal stages (Kim et al., 2018; Yin et al., 2019). Given the expansion of EGL in the P0 Sufu-cKO cerebellum, we wanted to examine whether $\mathrm{SHH}$ signaling activity is already altered in the newborn cerebellum driving further aberrant developmental steps. Examination of Gli protein levels in the P0 control and Sufu-cKO cerebellum show marked reduction of Gli1, Gli2, Gli3, and Ptch1 levels (Supplementary Fig. 1A); this is inconsistent with elevated SHH signaling. To directly examine this in specific EGL regions, we compared the cerebellum of PO control and Sufu-cKO mice carrying the SHH reporter transgene Gli1-LacZ(Ahn and Joyner, 2005). In these mice, SHH signaling activity is absent or very low throughout the entire P0 Sufu-cKO cerebellum but is highly active in a region-specific manner in controls (Fig. 4A). Furthermore, while some LacZ+ cells are detectable in EGL regions $A$ and $C$ and adjacent ML and IGL, LacZ+ cells are completely absent in EGL region B and adjacent areas of the P0 Sufu-cKO cerebellum (Fig. 4A; Supplementary Fig. 1B). These findings indicate that the accumulation of GNPs does not rely on active SHH signaling, particularly in Region B where there is a severe expansion of GNPs.

We previously observed that Sufu dysfunction in neocortical progenitors upregulates FGF ligand expression and subsequent activation of FGF signaling to influence lineage progression (Yabut et al., 2020). We therefore postulated that FGF signaling may similarly affect GNP behavior in the newborn cerebellum of Sufu-cKO mice. Since spatially distinct alterations in GNP expansion and cellular profiles are present in the P0 Sufu-cKO cerebellum, FGF ligands with immediate effect on GNP behavior will be exp ressed locally. Thus, we examined the spatial expression profiles of all FGF ligands (Ornitz and Itoh, 2015) in the late embryonic/early neonatal mouse cerebellum via the Allen Developing Mouse Brain Atlas (2008) (www.brain-map.org). This search revealed that expression of FGF5 specifically localizes to the secondary fissure of the developing cerebellum of the E18.5 wildtype mouse. In situ hybridization (ISH) using FGF5specific riboprobes confirms high expression of FGF5 (FGF5 high) immediately adjacent to the secondary fissure in the P0 control cerebellum (Fig. 4B). Strikingly, in the P0 Sufu-cKO cerebellum, there is anterior expansion of FGF5 ${ }^{\text {high }}$ expression regions (outlines, Fig. 4B), coinciding with areas near the secondary fissure where GNP expansion is most severe (Fig. 2A, 2D). Further, while FGF5expressing (FGF5+) cells are largely excluded from the EGL of the control cerebellum, a substantial number of FGF5+ cells are ectopically localized in the EGL Region B of the Sufu-cKO cerebellum (Fig. 4C). FGF5 mRNA molecules are visibly higher in FGF5-expressing cells in the Sufu-cKO EGL compared to controls (arrows, Fig. 4C). These findings 
bioRxiv preprint doi: https://doi.org/10.1101/2021.06.23.449636; this version posted June 24,2021 . The copyright holder for this preprint (which was not certified by peer review) is the author/funder, who has granted bioRxiv a license to display the preprint in perpetuity. It is made available under aCC-BY-NC-ND 4.0 International license.

Aberrant FGF signaling in SHH subgroup infantile medulloblastoma

A

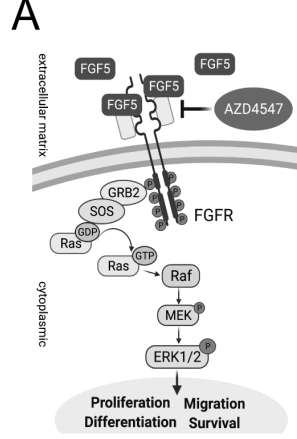

$B$ control Sufu-cKO

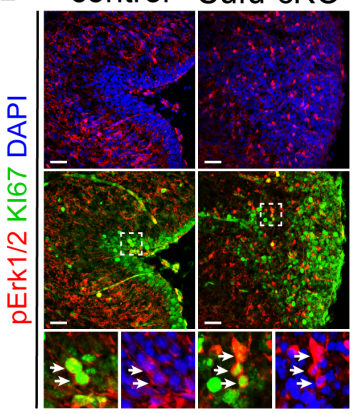

C
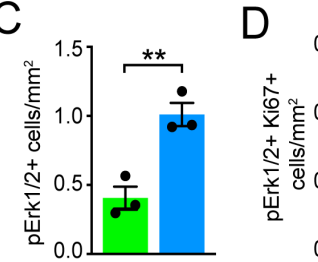

G
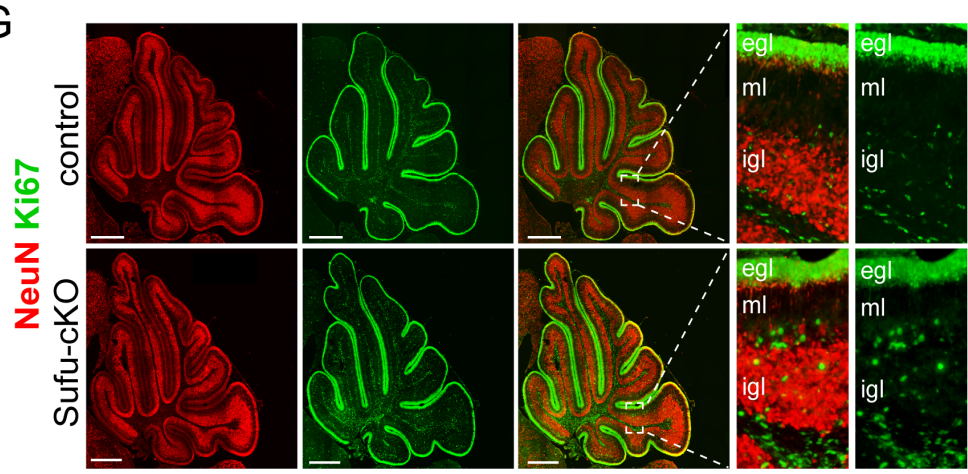

Figure 5: Ectopic activation of FGF signaling in the EGL of PO Sufu-cKO cerebellum. (A) Schematic diagram showing the activation of FGF signaling activity, upon binding of FGF5 to extracellular domains of FGFR, via the MAPK signal transduction pathway. (B) Doubleimmunofluorescence staining with Ki67 (green) and phospho-Erk1/2 (pErk1/2; red), a marker of activated MAPK signaling in the PO Sufu-cKO and control cerebelli. Boxed regions show pErk1/2+ and Ki67+ cells (arrowheads) in the control and Sufu-cKO EGL. Scale bars $=50 \mu \mathrm{m}$. (C, D) Quantification of pErk1/2+ cells (C) and double-labeled pErk1/2+ and Ki67+ cells (D) in the PO Sufu-cKO and control EGL Region B. ${ }^{* *} p<0.01$. (E) Experimental design of rescue studies performed by intraventricular administration of FGFR1-3 pharmacological inhibitor, AZD4547 or vehicle controls. (F) Nissl staining of the P7 control and Sufu-cKO treated with either AZD4547 or vehicle, 2 days after treatment. Scale bars $=500 \mu \mathrm{m}$. (G) NeuN and Ki67 double immunofluorescence staining of the P7 control and Sufu-cKO treated with either AZD4547 or vehicle. Boxed regions show localization and organization of $\mathrm{NeuN}+$ and $\mathrm{Ki67+}$ cells in distinct cerebellar layers. Arrows point to areas of the EGL and IGL where NeuN+ cells are beginning to be expressed.

implicate FGF5 as a potential instigator of region-specific defects in GNP differentiation present in the P0 Sufu-cKO cerebellum.

FGF signaling drives GNP proliferation in the PO Sufu-cKO cerebellum

FGF5 is a ligand for fibroblast growth factor receptors 1 (FGFR1) and 2 (FGFR2), both of which are expressed in the developing cerebellum, particularly in IGL regions where FGF5-expressing cells localize (Clements et al., 1993; Ornitz et al., 1996). Binding of FGF5 to these receptors triggers the activation of multiple intracellular signaling pathways including the mitogen activated protein kinase (MAPK) pathway to control cellular activities driving NSC progression (Fig. 5A) (Ornitz and Itoh, 2015). Immunostaining with antibodies to detect MAPK pathway activation reveals increased MAPK activity in the EGL of the P0 Sufu-cKO cerebellum. Regional distribution of cells labeled with phospho-Erk1/2 (pErk1/2+), a marker for

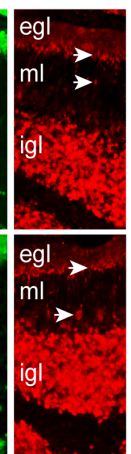

activated MAPK signaling, shows that the abnormally expanded EGL of the secondary fissure in the PO Sufu-cKO cerebellum has increases in these cells (Fig. 5B) and quantification of the number of pERK1/2+ cells in this area reveals that this is significant compared to controls (Fig. 5C). Many of these pERK1/2+ cells are also proliferative as indicated by Ki67 labeling (boxed areas, Fig. 5B) and the numbers of these dual labeled cells are significantly higher in the Sufu-cKO cerebellum compared to controls (Fig. 5D). These findings indicate that the increase in FGF5 correlates with the activation of FGF signaling in GNPs and demonstrates a likely role in regulating the abnormal proliferation and pre-neoplastic lesion in mutant mice.

Blockade of FGF signaling dramatically rescues the Sufu-cKO phenotype

To determine if activated FGF signaling drives GNP defects in the Sufu-cKO cerebellum, we pharmacologically inhibited FGF signaling using the competitive FGFR1-3 antagonist AZD4547 (Gudernova et al., 2016). For this experiment, 1 ul of AZD4547 $(5 \mathrm{mg} / \mathrm{ml})$ was delivered via intraventricular (IV) injection for 5 consecutive days beginning at $\mathrm{P} 0$ and the cerebellum analyzed 3 days after treatment at P7 (Fig. 5E). Strikingly, AZD4547 treatment results in near complete rescue of the GNP phenotype by P7 in the Sufu-cKO cerebellum, displaying a cerebellar morphology indistinguishable from controls with normal foliation and cellular organization (Fig. 5F). Indeed, in the cerebellum of AZD4547 -treated P7 Sufu-cKO mice, proliferating Ki67+ cells largely exclusively localize in the EGL while $\mathrm{NeuN}+$ cells densely pack the IGL and not the EGL (Fig. 5G). Notably, NeuN expression appears in cells localized at the border of the EGL and ML, where Ki67+ cells are absent, indicating that post-mitotic cells successfully began differentiation as observed in controls (boxed regions, Fig. 5G). Thus, our findings confirm that inhibition of FGF signaling in proliferating GNPs of the Sufu-cKO cerebellum ensure normal progression of GNP differentiation.

FGF5 expression is increased in the developing cerebellum of Sufu;p53-dKO mice

Our findings indicate a critical role for FGF signaling in driving GNP hyperplasia, making GNPs vulnerable to neoplastic lesions resulting in tumorigenesis, when Sufu is absent in the developing cerebellum. We sought to confirm that upregulated FGF signaling also occur in the tumorprone Sufu;p53-dKO. As expected, the neonatal cerebellum of Sufu-cKO, display EGL expansion due to excess 


\section{Aberrant FGF signaling in SHH subgroup infantile medulloblastoma}
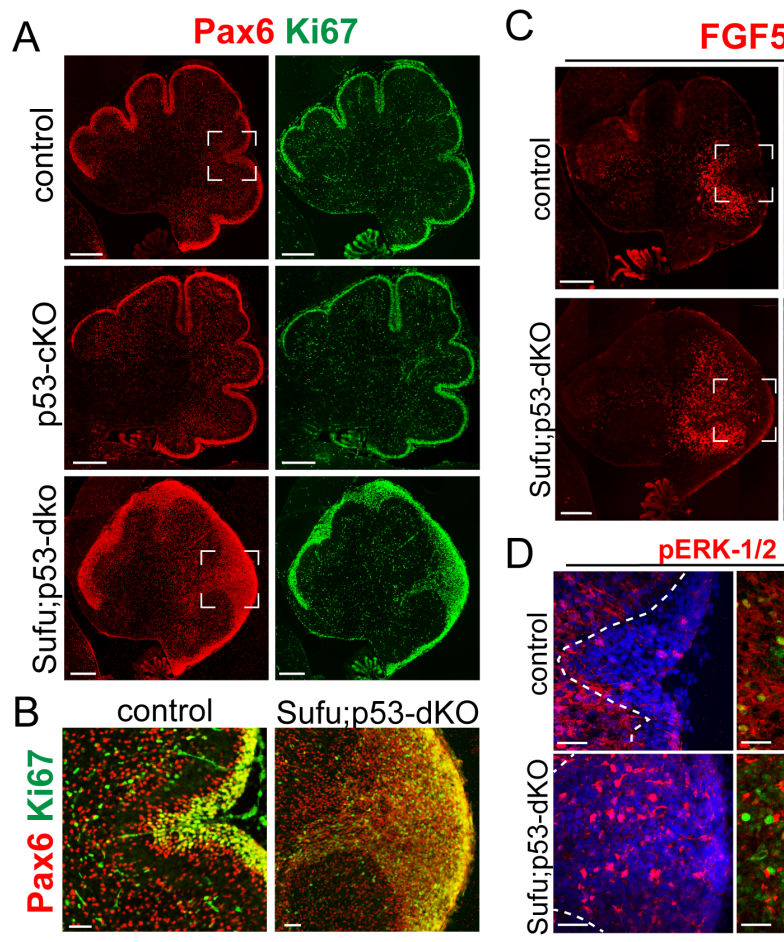

FGF5 DAPI
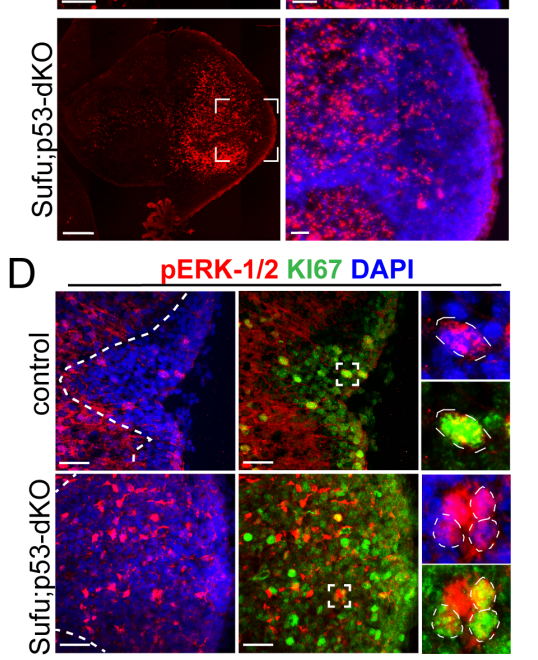

Figure 6: Elevated FGF5 expression correlates with activated FGF signaling in the developing cerebellum of P0 Sufu;p53-dKO mice. (A, B) Doubleimmunofluorescence staining against Pax6 (red) and Ki67 (green) in the P0 control, p53-cKO, and Sufu;p53-dKO cerebellum (A). Boxed regions in A are magnified in B demonstrating the expansion of the EGL in the PO Sufu;p53-dKO cerebellum compared to littermate controls. Scale bars $=200 \mu \mathrm{m}(\mathrm{A})$ and $50 \mu \mathrm{m}$ (B). (C) Fluorescent in situ hybridization using RNAScope probes against FGF5 mRNA (red) and DAPI labeling in the P0 Sufu;p53-dKO and control cerebellum. Boxed areas are enlarged to show ectopic localization of FGF5+ cells in the EGL of Sufu-p53-dKO cerebellum unlike in controls. Scale bars $=200 \mu \mathrm{m}$ and $50 \mu \mathrm{m}$ (boxed area). (D) Double-immunofluorescence staining with Ki67 (green) and phospho-Erk1/2 (pErk1/2; red) in the P0 Sufu;p53-dKO and control cerebelli. Boxed regions show cells double-labeled with pErk1/2+ and Ki67+ cells in the control and Sufu;p53-dKO EGL Region B. Scale bars $=25 \mu \mathrm{m}$.

proliferative (Ki67+) and Pax6+ cells in the P0 Sufu;p53dKO cerebellum but not in p53-cKO and control cerebelli (Fig. 6A). Further expansion of Pax6+ GNPs in the P0 Sufu;p53-dKO is also most severe along the secondary fissure (EGL Region B) compared to other EGL areas (EGL Regions A and C) of the P0 Sufu;p53-dk0 cerebellum (Fig. 6A-6B). As with our observations in the PO Sufu-cKO cerebellum, ISH for FGF5 in the P0 Sufu;p53-dKO cerebellum show ectopic FGF5 expression. Particularly, FGF5+ cells are expanded anteriorly and detected specifically around the secondary fissure of the P0 Sufu;p53-dKO cerebellum (Fig. 6C). There is also ectopic MAPK signaling activity in the P0 Sufu;p53-dKO cerebellum, with significantly higher numbers of pErk $1 / 2+$ cells within the EGL, many of which are proliferative as marked by colabeling with Ki-67, within the expanded EGL of the secondary fissure (Fig. 6D). These findings indicate that as in the P0 Sufu-cKO cerebellum, ectopic FGF5 expression triggers FGF signaling in GNPs in the P0 Sufu;p53-dKO cerebellum and may facilitate oncogenic transformation of GNPs.

FGF5 is specifically upregulated in SHH-driven infantile MB. Given the critical role of the FGF signaling axis in driving GNP expansion in the neonatal Sufu-cKO and Sufu;p53-dKO cerebellar phenotypes, we sought to determine if key FGF signaling pathway genes are differentially expressed in MB patient tumors. We performed comparative analysis of the expression dataset from $763 \mathrm{MB}$ patient samples comprised of tumors resected from $223 \mathrm{MB}^{\mathrm{SHH}}, 70 \mathrm{MB}$ WNT, $144 \mathrm{MB}^{\text {Group3}}$, and 326 MBGroup4 patients (Cavalli et al., 2017). Strikingly, our analyses show that FGF5 expression is higher in tumors specifically from $\mathrm{MB}^{\mathrm{SHH}}$ patients compared to other MB subgroups, with approximately $25 \%$ of $\mathrm{MB}^{\mathrm{SHH}}$ tumors exhibiting a two-fold increase (Fig. 7A-7B). We also find that FGF5 is uniquely upregulated in $\mathrm{MB}^{\text {SHH }}$ tumors from patients within the 0-3-year-old age group, but not patients within the 0-3-year-old age group in other MB subtypes (Fig. 7C). Further examination across all $\mathrm{MB}^{\mathrm{SHH}}$ tumors stratified which subgroups express the highest levels of FGF5 expression. Infantile tumors, largely belonging within the SHHb and SHHg subgroups (Cavalli et al., 2017), exhibit higher FGF5 levels compared to tumors from children (SHHa) or adults (SHHd) (Fig. 7D). By all measures, the proportion of SHHb and SHHg tumors with relatively high levels of FGF5 is significantly increased $(\sim 30 \%)$ compared to other $\mathrm{MB}^{\mathrm{SHH}}$ subgroups (Fig. 7E). Taken together, these findings strongly suggest that FGF signaling is specifically disrupted in infantile-onset $\mathrm{MB}^{\mathrm{SHH}}$.

\section{Discussion}

Infants harboring mutations in Suppressor of Fused comprise $\sim 30 \%$ of patients with $\mathrm{MB}^{\mathrm{SHH}}$. These patients have worse prognosis, higher rates of local recurrence, and do not readily respond to available therapeutics specifically targeting SHH signaling (Guerrini-Rousseau et al., 2018; Kool et al., 2014; Schwalbe et al., 2017). We postulated that the combinatorial effects of oncogenic SUFU mutations and other concurrent developmental signaling pathways, make GNPs vulnerable to oncogenic transformation leading to infantile $\mathrm{MB}^{\mathrm{SH}}$. Using mice lacking Sufu in granule neuron lineages, the studies here identified region-specific FGF signaling as a driver of oncogenesis in infantile MBSнH. Specifically, ectopic FGF5 gene expression in defined regions triggers FGF signaling in proliferating GNPs resulting in GNP hyperplasia and patterning defects. Inhibition of FGF signaling through pharmacological blockade of FGFR1-3 prevents hyperplasia and associated cerebellar architectural abnormalities. We further found that elevated levels of FGF5 gene expression in infantile $\mathrm{MB}^{\mathrm{SHH}}$ patients, but not in other MB subgroups. These findings indicate that FGF-targeting compounds are likely to be a promising therapeutic option for infantile $\mathrm{MB}^{\mathrm{SHH}}$ patients, particularly those marked by upregulated FGF5 and FGFR signaling.

Sufu targets modulators of granule neuron lineage progression in spatially distinct neurogenic zones of the neonatal cerebellum.

The EGL of the newborn Sufu-cKO cerebellum is very expanded and packed with Pax6+ GNPs, but the severity of GNP expansion differs regionally and matches the anatomic subregions where infantile MB typically arises. In the wildtype cerebellum, there are low levels of Gli1 reporter 
A
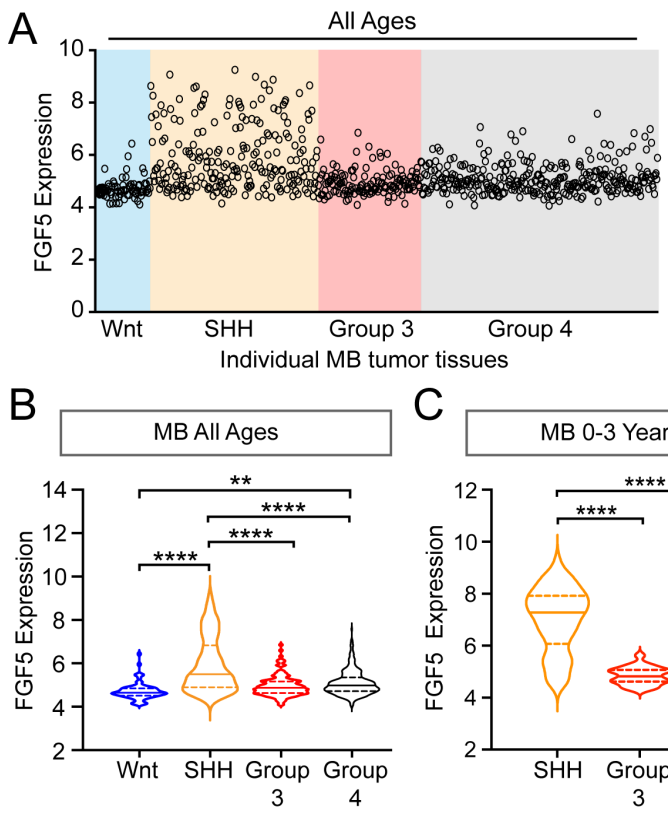
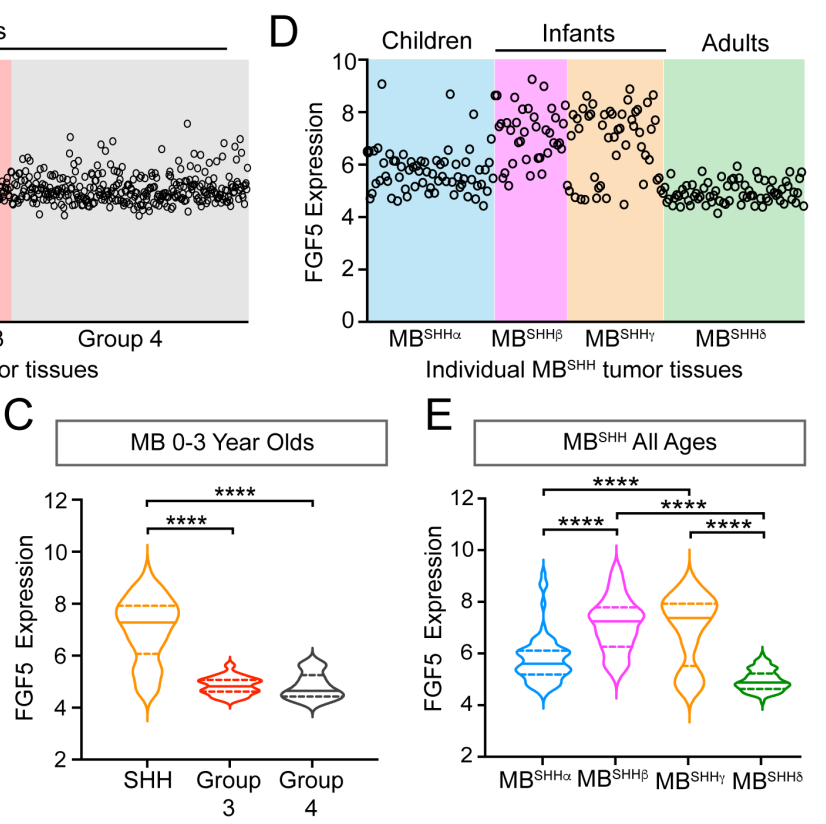

Figure 7: Upregulated FGF5 expression in MBSHH tumors from infant patients. (A) Levels of FGF5 expression in human MB tumors of all ages from GEO expression dataset \#GSE85217(Cavalli et al., 2017). (B, C) Statistical analysis of FGF5 expression levels associated with MB tumor subtypes from patients across all ages (B) and 0-3 year old MB patients (C). ${ }^{* *} p<0.01,{ }^{* \star * *} p<0.0001$. (D, E) Graph represents FGF5 expression levels in human MBSHH tumors of all ages from GEO expression dataset \#GSE85217 (D) and corresponding plots (E) showing statistically higher FGF5 expression in tumors from infants with $\mathrm{MB}^{\mathrm{SHH}}$ compared to tumors from children or adults with $\mathrm{MB}^{\mathrm{SHH}}{ }^{* \star * *} \mathrm{p}<0.0001$.

activity, Ptch1 expression, and SHH ligand expression in this subregion (Corrales et al., 2004). This suggest important roles of non-canonical SHH signaling activity beyond Smo, under Sufu control, in regulating granule neuron development in this area. Indeed, over the course of neonatal cerebellar development, the Sufu-cKO cerebellum has persistent GNP proliferation, and failure to produce mature granule neurons. Future studies must elucidate further signaling mechanisms for Sufu that control the timely exit from the cell cycle and promote the continued differentiation of post-mitotic GNPs.

Sufu dysfunction leads to alterations in FGF signaling in various neurogenic regions of the embryonic brain. Under these conditions, loss of Sufu results in the destabilization and eventual degradation of Gli transcription factors leading to deregulated expression of Gli-dependent target genes (Chen et al., 2009; Humke et al., 2010). Ectopic expression of FGF ligands, FGF8 and FGF15, have been associated with degradation of Gli3R as a consequence of Sufu deletion (Jiwani et al., 2020; Kim et al., 2011, 2018; Yabut et al., 2020). Increases in FGF levels result in defects in regional patterning and progenitor specification and differentiation. In the postnatal cerebellum, we found ectopic expression of FGF5. FGF5 is a secreted ligand capable of binding to FGFR1-3 receptors, which transduce signals intracellularly via MAPK or PI3K signaling(Ornitz and Itoh, 2015). Confirming the functional signaling role of this ectopic FGF5, we find excess regional activation of both MAPK signaling in the P0 Sufu-cKO cerebellum (Fig. 8).

The increase in FGF5 gene expression is associated with reduced levels of Gli1, Gli2, and Gli3 (Fig. 8). Thus, while we postulate that Gli3R is the likely direct repressor of FGF5 in line with previous studies, we cannot yet eliminate the possibility that FGF5 is repressed indirectly by Gli1 or Gli2 gene targets or other unidentified molecular targets of Sufu. Nevertheless, the identification of FGF5 as a downstream gene target following loss of Sufu in the developing cerebellum indicate that Sufu acts at the intersection of $\mathrm{SHH}$ and $\mathrm{FGF}$ signaling to modulate precursor behavior. These findings build on previous studies and establish Sufu as critical for ensuring lineage progression of progenitor populations in neurogenic regions. In the cerebellum, disrupting Sufu function can therefore make GNPs vulnerable to oncogenic insults driving $\mathrm{MB}$ formation.

\section{Lineage progression of cerebellar granule neurons rely on strict modulation of FGF signaling.}

Previous studies of FGF signaling showed important functions in cerebellar granule neuron development that are reminiscent of phenotypes in mice lacking Sufu. Combined loss of FGFR1 and FGFR2 in GNPs at late stages of embryonic cerebellar development lead to perturbations in cerebellar organization and granule neuron production (Smith et al., 2012). As with Sufu-deficient mice, conditional ablation of single or multiple FGFRs in GNPs at early neonatal stages do not result in severe cerebellar defects (Emmenegger et al., 2013; Jiwani et al., 2020; Yin et al., 2019). We believe these similarities are driven by the embryonic regulatory function of Sufu on FGF signaling (Jiwani et al., 2020; Kim et al., 2018; Yabut et al., 2020). In the wildtype cerebellum, it is striking that FGF5-expressing cells localize within the IGL surrounding the secondary fissure at P0 (Fig. 4B) (Hattori et al., 1997; Ozawa et al., 1996; Yaguchi et al., 2009). As mentioned above, these areas also display the lowest Gli1 reporter activity, Ptch1 expression, and SHH ligand expression (Fig. 4A) (Corrales et al., 2004). By P4, FGF5 expression is detected in cells within the IGL throughout the cerebellum (Yaguchi et al., 2009). However, by P14, a timepoint at which most granule neurons have differentiated, FGF5 expression is no longer detected in the IGL or other cerebellar regions (data not shown) (Lein et al., 2007). The strict spatiotemporal expression of FGF5 strongly indicates a stage-specific role for regulating granule neuron lineage progression. Indeed, the ectopic expansion of FGF5-expressing cells in the Sufu-cKO cerebellum is associated with the expansion of the adjacent EGL where misdifferentiated GNPs localize. Importantly, these phenotypes are significantly alleviated after pharmacological inhibition of FGFRs. Thus, the presence of FGF5 along the secondary fissure must trigger FGF signaling to induce timely differentiation and maturation of granule neurons. Further studies will identify FGF5-responsive cells and elucidate if and how FGF5 exerts autocrine or paracrine functions to influence granule neuron lineage progression in the developing cerebellum. 


\section{Aberrant FGF signaling in SHH subgroup infantile medulloblastoma}

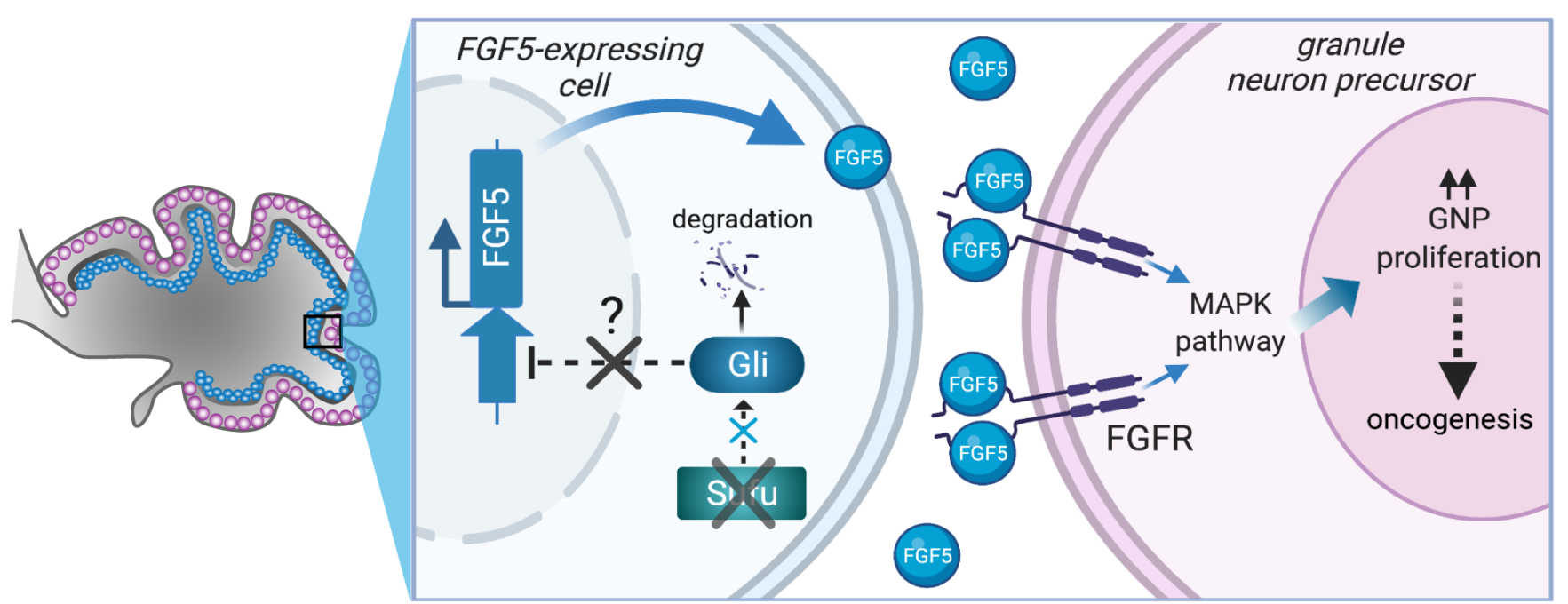

Figure 8: Loss of Sufu function drives excess proliferation of granule neuron precursors via FGF signaling activation. The schematic diagram summarizes how Sufu LOF facilitate expansion of GNPs at early stages of cerebellar development by driving FGF signaling activation via ectopic expression of FGF5.

Our findings are contrary to previous reports of the proliferation-suppressive roles of FGFs in GNPs specifically carrying Ptch1 mutations (Emmenegger et al., 2013; Fogarty et al., 2007). However, distinct mechanisms are at play downstream of SHH signaling in mice carrying Ptch1 or Sufu mutations. In contrast to the Ptch1-cK0 cerebellum, the newborn Sufu-cKO cerebellum expresses Ptch1 and protein levels of Gli1, Gli2, and are decreased (Supplementary Fig. 1A). These key differences may activate unique networks downstream of SHH signaling in GNPs and surrounding cells in the Sufu-cKO cerebellum. Additionally, there are approximately 18 FGF ligands which differ significantly in molecular features and binding specificities to distinct combinations of FGFR splice variants(Ornitz and Itoh, 2015). For example, unlike FGF2, FGF5 can exert both autocrine and paracrine functions, bind different combinations of FGFRs, and is dynamically expressed in distinct regions of the developing cerebellum. Thus, in-depth studies are needed to elucidate the FGFR and potentially non-FGFR signaling mechanisms triggered by abnormally high levels of FGF5 in the developing cerebellum, particularly since FGF5 overexpression is known to drive cancer development and progression, including brain tumors (Allerstorfer et al., 2008).

FGF5 expression and FGF signaling activity meet criteria as key drivers of infantile medulloblastoma.

Infantile MBs associated with Sufu mutations are common indicating the selective developmental vulnerability of the neonatal cerebellum to neoplastic effects of Sufu dysfunction. We have identified specific cellular and molecular mechanisms disrupted by Sufu LOF facilitating GNP transformation. Furthermore, we conclude that the timing of Sufu LOF is critical; conditional deletion of Sufu in neural stem cells prior to granule neuron specification (using the hGFAP-Cre line) results in GNP hyperplasia, whereas conditional deletion of Sufu after granule neuron specification (using the Math1-Cre line) does not lead to these defects (Jiwani et al., 2020). Thus, Sufu-associated infantile MB is a likely consequence of defects stemming from early stages of granule neuron lineage specification at embryonic stages.
Tumors initiated at embryonic stages are typically undetectable until several months after birth when tumorigenesis has significantly progressed. Thus, therapeutics for infantile MB must specifically target mechanisms driving tumorigenesis at this postnatal stage and avoid effects on normal GNPs elsewhere in the cerebellum undergoing normal proliferation, differentiation, and establishing connectivity at these stages. Our successful identification of excessive FGF5 and FGF signaling activity as a consequence of Sufu LOF provide new paths toward the design of targeted treatments for infantile MB. Since we confirmed that high levels of FGF5 occur uniquely in a subset of infantile $\mathrm{MB}^{\mathrm{SHH}}$ patients, FGF5 expression levels may be useful as a diagnostic biomarker for $\mathrm{MB}^{\mathrm{SHH}}$ patients to test drugs specifically targeting FGF signaling. There is also a need to determine whether specifically targeting FGF5 or its cognate receptors FGFR1/FGFR2 is sufficient to induce an anti-tumor response. Ultimately, we hope that these studies will facilitate the design of therapies specifically targeting oncogenic mechanisms in $\mathrm{MB}^{\mathrm{SHH}}$ without significant disruption of normal developmental processes.

\section{Acknowledgements}

We thank Hirofumi Noguchi and other members of the Pleasure Lab for critical discussions, the UCSF Center for Advanced Light Microscopy for assistance with imaging, and William Krause for assistance with transcriptomics. Schematic diagrams were created with BioRender.com. This paper was typeset with the bioRxiv word template by @Chrelli: www.github.com/chrelli/bioRxiv-word-template. This work was supported by the NIH R01MH077694 and R01NS118995 (S.J.P.), R01MH077694-S1 (H.G.), NIH NIH/NCI K01CA201068 (O.R.Y.), and American Brain Tumor Association Grant \#A131363 (O.R.Y.).

\section{Competing interest statement}

The authors declare no competing financial interest.

\section{References}

Ahn S, Joyner AL. 2005. In vivo analysis of quiescent adult neural stem cells responding to Sonic hedgehog. Nature 437:894-897. doi:10.1038/nature03994

Allerstorfer S, Sonvilla G, Fischer H, Spiegl-Kreinecker S, Gauglhofer C, Setinek U, Czech T, Marosi C, Buchroithner J, Pichler J, et al. 2008. FGF5 as an oncogenic factor in human glioblastoma multiforme: autocrine and paracrine activities. Oncogene 27:4180-4190. doi:10.1038/onc.2008.61 
bioRxiv preprint doi: https://doi.org/10.1101/2021.06.23.449636; this version posted June 24,2021 . The copyright holder for this preprint (which was not certified by peer review) is the author/funder, who has granted bioRxiv a license to display the preprint in perpetuity. It is made available under aCC-BY-NC-ND 4.0 International license.

\section{Aberrant FGF signaling in SHH subgroup infantile medulloblastoma}

Barrett T, Wilhite SE, Ledoux P, Evangelista C, Kim IF, Tomashevsky M, Marshall KA, Phillippy KH, Sherman PM, Holko M, et al. 2013. NCBI GEO: archive for functional genomics data sets--update. Nucleic Acids Res 41:D991-5. doi:10.1093/nar/gks1193

Cavalli FMG, Remke M, Rampasek L, Peacock J, Shih DJH, Luu B, Garzia L, Torchia J, Nor C, Morrissy AS, Agnihotri S, Taylor MD, et al. 2017. Intertumoral Heterogeneity within Medulloblastoma Subgroups. Cancer Cell 31:737-754.e6. doi:10.1016/j.ccell.2017.05.005

Chen M-H, Wilson CW, Li Y-J, Law KK Lo, Lu C-S, Gacayan R, Zhang X, Hui C, Chuang P-T. 2009. Cilium-independent regulation of Gli protein function by Sufu in Hedgehog signaling is evolutionarily conserved. Genes Dev 23:1910-28. doi:10.1101/gad.1794109

Clements DA, Wang JK, Dionne CA, Goldfarb M. 1993. Activation of fibroblast growth factor (FGF) receptors by recombinant human FGF-5. Oncogene 8:1311-6.

Corrales JD, Rocco GL, Blaess S, Guo Q, Joyner AL. 2004. Spatial pattern of sonic hedgehog signaling through Gli genes during cerebellum development. Development 131:5581-90. doi:10.1242/dev.01438

Emmenegger BA, Hwang EI, Moore C, Markant SL, Brun SN, Dutton JW, Read T-A, Fogarty MP, Singh AR, Durden DL, et al. 2013. Distinct roles for fibroblast growth factor signaling in cerebellar development and medulloblastoma. Oncogene 32:4181-4188. doi:10.1038/onc.2012.440

Fogarty MP, Emmenegger BA, Grasfeder LL, Oliver TG, Wechsler-Reya RJ, Lefkowitz RJ. 2007. Fibroblast growth factor blocks Sonic hedgehog signaling in neuronal precursors and tumor cells.

Frappart P-O, Lee Y, Russell HR, Chalhoub N, Wang Y-D, Orii KE, Zhao J, Kondo N, Baker SJ, McKinnon PJ. 2009. Recurrent genomic alterations characterize medulloblastoma arising from DNA double-strand break repair deficiency. Proc Natl Acad Sci U S A 106:1880-5. doi:10.1073/pnas.0806882106

Gao Y, Ferguson DO, Xie W, Manis JP, Sekiguchi J, Frank KM, Chaudhuri J, Horner J, DePinho RA, Alt FW. 2000. Interplay of p53 and DNA-repair protein XRCC4 in tumorigenesis, genomic stability and development. Nature 404:897-900. doi:10.1038/35009138

Gudernova I, Vesela I, Balek L, Buchtova M, Dosedelova H, Kunova M, Pivnicka J, Jelinkova I, Roubalova L, Kozubik A, Krejci P. 2016. Multikinase activity of fibroblast growth factor receptor (FGFR) inhibitors SU5402, PD173074, AZD1480, AZD4547 and BGJ398 compromises the use of small chemicals targeting FGFR catalytic activity for therapy of short-stature syndromes. Hum Mol Genet 25:9-23. doi:10.1093/hmg/ddv441

Guerrini-Rousseau L, Dufour C, Varlet P, Masliah-Planchon J, Bourdeaut F, Guillaud-Bataille M, Abbas R, Bertozzi A-I, Fouyssac F, et al. 2018. Germline SUFU mutation carriers and medulloblastoma: clinical characteristics, cancer risk, and prognosis. Neuro Oncol 20:1122-1132. doi:10.1093/neuonc/nox228

Hattori Y, Miyake A, Mikami T, Ohta M, Itoh N. 1997. Transient expression of FGF-5 mRNA in the rat cerebellar cortex during post-natal development. Mol Brain Res 47:262-266. doi:10.1016/S0169-328X(97)00065-X

Humke EW, Dorn K V., Milenkovic L, Scott MP, Rohatgi R. 2010. The output of Hedgehog signaling is controlled by the dynamic association between Suppressor of Fused and the Gli proteins. Genes Dev 24:670-82. doi:10.1101/gad.1902910

Jiwani T, Kim JJ, Rosenblum ND. 2020. Suppressor of fused controls cerebellum granule cell proliferation by suppressing Fgf8 and spatially regulating Gli proteins. Development 147:dev170274. doi:10.1242/dev.170274

Kim JJ, Gill PS, Rotin L, van Eede M, Henkelman RM, Hui C-C, Rosenblum ND. 2011. Suppressor of fused controls mid-hindbrain patterning and cerebellar morphogenesis via GLI3 repressor. J Neurosci 31:1825-36. doi:10.1523/JNEUROSCI.2166-10.2011

Kim JJ, Jiwani T, Erwood S, Loree J, Rosenblum ND. 2018. Suppressor of fused controls cerebellar neuronal differentiation in a manner modulated by GLI3 repressor and Fgf15. Dev Dyn 247:156-169. doi:10.1002/dvdy.24526

Kong JH, Siebold C, Rohatgi R. 2019. Biochemical mechanisms of vertebrate hedgehog signaling. Development 146. doi:10.1242/dev.166892
Kool M, Jones DTW, Jäger N, Northcott PA, Pugh TJ, Hovestadt V, Piro RM, Esparza LA, Markant SL, Remke M, Milde T, ICGC PedBrain Tumor Project, et al. 2014. Genome Sequencing of SHH Medulloblastoma Predicts Genotype-Related Response to Smoothened Inhibition. Cancer Cell 25:393-405. doi:10.1016/j.ccr.2014.02.004

Kool M, Korshunov A, Remke M, Jones DTW, Schlanstein M, Northcott PA, Cho Y-J, Koster J, Schouten-van Meeteren A, et al. 2012. Molecular subgroups of medulloblastoma: an international meta-analysis of transcriptome, genetic aberrations, and clinical data of WNT, SHH, Group 3, and Group 4 medulloblastomas. Acta Neuropathol 123:473-484. doi:10.1007/s00401-012-0958-8

Lein ES, Hawrylycz MJ, Ao N, Ayres M, Bensinger A, Bernard A, Boe AF, Boguski MS, Brockway KS, Byrnes EJ, Chen L, Jones AR, et al. 2007. Genomewide atlas of gene expression in the adult mouse brain. Nature 445:168176. doi:10.1038/nature 05453

Leto K, Arancillo M, Becker EBE, Buffo A, Chiang C, Ding B, Dobyns WB, Dusart I, Haldipur P, Hatten ME, et al. 2016. Consensus Paper: Cerebellar Development. The Cerebellum 15:789-828. doi:10.1007/s12311-0150724-2

Mah L-J, El-Osta A, Karagiannis TC. 2010. $\gamma \mathrm{H} 2 \mathrm{AX}$ : a sensitive molecular marker of DNA damage and repair. Leukemia 24:679-686. doi:10.1038/leu.2010.6

Ornitz DM, Itoh N. 2015. The Fibroblast Growth Factor signaling pathway. Wiley Interdiscip Rev Dev Biol 4:215-66. doi:10.1002/wdev.176

Ornitz DM, Xu J, Colvin JS, McEwen DG, MacArthur CA, Coulier F, Gao G, Goldfarb M. 1996. Receptor specificity of the fibroblast growth factor family. J Biol Chem 271:15292-7. doi:10.1074/jbc.271.25.15292

Ostrom QT, Gittleman H, Xu J, Kromer C, Wolinsky Y, Kruchko C, BarnholtzSloan JS. 2016. CBTRUS Statistical Report: Primary Brain and Other Central Nervous System Tumors Diagnosed in the United States in 2009-2013. Neuro Oncol 18:v1-v75. doi:10.1093/neuonc/now207

Ozawa K, Uruno T, Miyakawa K, Seo M, Imamura T. 1996. Expression of the fibroblast growth factor family and their receptor family genes during mouse brain development. Mol Brain Res 41:279-288. doi:10.1016/0169328X(96)00108-8

Pazzaglia S, Tanori M, Mancuso M, Rebessi S, Leonardi S, Di Majo V, Covelli V, Atkinson MJ, Hahn H, Saran A. 2006. Linking DNA damage to medulloblastoma tumorigenesis in patched heterozygous knockout mice. Oncogene 25:1165-1173. doi:10.1038/sj.onc.1209032

Pospisilik JA, Schramek D, Schnidar H, Cronin SJF, Nehme NT, Zhang X, Knauf C, Cani PD, Aumayr K, Todoric J, et al. 2010. Drosophila genome-wide obesity screen reveals hedgehog as a determinant of brown versus white adipose cell fate. Cell 140:148-60. doi:10.1016/j.cell.2009.12.027

Remke M, Ramaswamy V. 2018. Infant medulloblastoma - learning new lessons from old strata. Nat Rev Clin Oncol 15:659-660. doi:10.1038/s41571-018-0071-6

Schwalbe EC, Lindsey JC, Nakjang S, Crosier S, Smith AJ, Hicks D, Rafiee G, Hill RM, Iliasova A, Stone T, et al. 2017. Novel molecular subgroups for clinical classification and outcome prediction in childhood medulloblastoma: A cohort study. Lancet Oncol 18:958-971. doi:10.1016/S1470-2045(17)30243-7

Smith KM, Williamson TL, Schwartz ML, Vaccarino FM. 2012. Impaired motor coordination and disrupted cerebellar architecture in Fgfr1 and Fgfr2 double knockout mice. Brain Res 1460:12-24. doi:10.1016/J.BRAINRES.2012.04.002

Tan I-L, Wojcinski A, Rallapalli H, Lao Z, Sanghrajka RM, Stephen D, Volkova E, Korshunov A, Remke M, Taylor MD, Turnbull DH, Joyner AL. 2018. Lateral cerebellum is preferentially sensitive to high sonic hedgehog signaling and medulloblastoma formation. Proc Natl Acad Sci U S A 115:3392-3397. doi:10.1073/pnas.1717815115

Vanner RJ, Remke M, Gallo M, Selvadurai HJ, Coutinho F, Lee L, Kushida M, Head R, Morrissy S, Zhu X, et al. 2014. Quiescent Sox2+ Cells Drive Hierarchical Growth and Relapse in Sonic Hedgehog Subgroup Medulloblastoma. Cancer Cell 26:33-47. doi:10.1016/j.ccr.2014.05.005

Vladoiu MC, El-Hamamy I, Donovan LK, Farooq H, Holgado BL, Sundaravadanam Y, Ramaswamy V, Hendrikse LD, Kumar S, Mack SC, et al. 
bioRxiv preprint doi: https://doi.org/10.1101/2021.06.23.449636; this version posted June 24,2021 . The copyright holder for this preprint (which was not certified by peer review) is the author/funder, who has granted bioRxiv a license to display the preprint in perpetuity. It is made available under aCC-BY-NC-ND 4.0 International license.

\section{Aberrant FGF signaling in SHH subgroup infantile medulloblastoma}

2019. Childhood cerebellar tumours mirror conserved fetal transcriptional programs. Nature 572:67-73. doi:10.1038/s41586-019-1158-7

Ward E, DeSantis C, Robbins A, Kohler B, Jemal A. 2014. Childhood and adolescent cancer statistics, 2014. CA Cancer J Clin 64:83-103. doi:10.3322/caac. 21219

Wechsler-Reya RJ, Scott MP. 1999. Control of Neuronal Precursor Proliferation in the Cerebellum by Sonic Hedgehog. Neuron 22:103-114. doi:10.1016/S0896-6273(00)80682-0

Wefers AK, Warmuth-Metz M, Pöschl J, von Bueren AO, Monoranu C-M, Seelos K, Peraud A, Tonn J-C, Koch A, et al. 2014. Subgroup-specific localization of human medulloblastoma based on pre-operative MRI. Acta Neuropathol 127:931-933. doi:10.1007/s00401-014-1271-5

Yabut OR, Fernandez G, Huynh T, Yoon K, Pleasure SJ. 2015. Suppressor of Fused Is Critical for Maintenance of Neuronal Progenitor Identity during Corticogenesis. Cell Rep 12:2021-2034. doi:10.1016/j.celrep.2015.08.031

Yabut OR, Ng H-X, Yoon K, Arela JC, Ngo T, Pleasure SJ. 2020. The neocortical progenitor specification program is established through combined modulation of $\mathrm{SHH}$ and FGF signaling. $J$ Neurosci. doi:10.1523/JNEUROSCI.2888-19.2020

Yaguchi Y, Yu T, Ahmed MU, Berry M, Mason I, Basson MA. 2009. Fibroblast growth factor (FGF) gene expression in the developing cerebellum suggests multiple roles for FGF signaling during cerebellar morphogenesis and development. Dev Dyn 238:2058-2072. doi:10.1002/dvdy.22013

Yin W-C, Satkunendran T, Mo R, Morrissy S, Zhang X, Huang ES, UuskülaReimand L, Hou H, Son JE, Liu W, et al. 2019. Dual Regulatory Functions of SUFU and Targetome of GLI2 in SHH Subgroup Medulloblastoma. Dev Cell 48:167-183.e5. doi:10.1016/j.devcel.2018.11.015

Zhuo L, Theis M, Alvarez-Maya I, Brenner M, Willecke K, Messing A. 2001. hGFAP-cre transgenic mice for manipulation of glial and neuronal function in vivo. Genesis 31:85-94.

\section{Materials and Methods \\ Animals}

Mice carrying the floxed Sufu allele (Sufufl) were kindly provided by Dr. Chi-Chung Hui (University of Toronto) and were genotyped as described elsewhere(Pospisilik et al., 2010). hGFAP-cre (Stock \#004600) and Gli1LacZ (Stock \#008211) mice were obtained from Jackson Laboratories (Bar Harbor, ME, USA). Mice designated as controls did not carry the Cre transgene and may have either one of the following genotypes: $\mathrm{Su} f \mathrm{ffl}^{\mathrm{f}+}$ or Sufufl/fl. All mouse lines were maintained in mixed strains, and analysis included male and female pups from each age group, although sex differences were not included in data reporting. All animal protocols were in accordance to the National Institute of Health regulations and approved by the UCSF Institutional Animal Care and Use Committee (IACUC).

\section{In vivo treatment with FGFR inhibitor}

The FGFR1-3 inhibitor, AZD4547 (Selleck Chemicals, \#S2801) was dissolved sequentially in 4\% Dimethyl Sulfoxide (DMSO), 30\% Polyethylene glycol (PEG), 5\% Tween-80, and water to make a $1 \mathrm{mM}$ solution. 1 ul of $1 \mathrm{mM}$ AZD 4547, or vehicle only as control, was injected into the lateral ventricle ( $\sim 1 \mathrm{~mm}$ from the cerebellar midline) of pups for 5 days from P0/P1 using a $2.5 \mathrm{ml}$ syringe (Model $62 \mathrm{RN}$; Hamilton Scientific). Pups remained with the mother until perfusion at $\mathrm{P} 7$ for analysis.

\section{Immunohistochemistry and LacZ Staining}

Perfusion, dissection, and immunofluorescence and LacZ staining were conducted according to standard protocols as previously described(Yabut et al., 2015). Briefly, P0/P1 brain tissues were fixed after dissection by direct immersion in $4 \%$ paraformaldehyde (PFA) and P7 and older postnatal brains fixed by intracardial perfusion followed by $2 \mathrm{~h}$ postfixation. All fixed brains were cryoprotected a 15-30\% sucrose gradient prior to cryosectioning. Cryostat sections were air dried and rinsed $3 \times$ in PBS plus $0.2 \%$ Triton before blocking for $1 \mathrm{~h}$ in $10 \%$ normal lamb serum diluted in PBS with $0.2 \%$ Triton to prevent nonspecific binding. A heatinduced antigen retrieval protocol was performed on selective immunohistochemistry using $10 \mathrm{M}$ Citric Acid at $\mathrm{pH}$ 6.0. Primary antibodies were diluted in $10 \%$ serum diluted in PBS with $0.2 \%$ Triton containing 4'6-diamidino-2-phenylindole (DAPI); sections were incubated in primary antibody overnight at room temperature. The following antibodies were used: rabbit anti-Pax6 (1:250 dilution; Cat. \#: 901301, Biolegend); rabbit anti-NeuN (1:250 dilution; Cat. \#: PA5-784-99, Invitrogen); mouse anti-Calretinin (1:250, Cat. \#: AB5054, Millipore); rabbit anti-phospho-Erk1/2 (1:250 dilution; Cat. \#: 4370, Cell Signaling); $\gamma \mathrm{H} 2 \mathrm{AX}$ (1:100 dilution; Cat. \#: 05-636, Millipore); mouse anti-Ki67 (1:100 dilution; Cat. \#: 550809 BD Biosciences) and cleaved-Caspase 3 (1:250 dilution; Cat. \#: 9661S, Cell Signaling). To detect primary antibodies, we used species-specific Alexa Fluor-conjugated secondary antibodies (1:500; Invitrogen) in $1 \mathrm{X}$ PBS-T for $1 \mathrm{~h}$ at room temperature, washed with $1 \mathrm{X}$ PBS, and coverslipped with Fluoromount-G (SouthernBiotech).

In Situ Hybridization RNAScope ISH was conducted for FGF15 and Ptch1. RNAscope probes for Mm-FGF5 were designed commercially by the manufacturer (Advanced Cell Diagnostics, Inc.). RNAScope Assay was performed using the RNAscope Multiplex Fluorescent Reagent Kit V2 according to manufacturer's instructions. Detection of the probe was done with Opal 570 or Opal 520 reagent (Akoya Biosciences).

\section{Western Blot Analysis}

Western blot analyses were conducted according to standard protocols. Soluble extracts were loaded onto Criterion, 4-15\% Tris-HCI 4 SDS-PAGE gels (Bio-Rad), separated at 120V, and transferred to PVDF membrane at $30 \mathrm{~V}$ for 2 hours or overnight at $4^{\circ} \mathrm{C}$. Membranes were blocked with $3 \%$ milk/1X TBS-T (Tris-buffered saline with $0.1 \%$ Tween 20 ) or $5 \%$ BSA/1X TBS-T for $1 \mathrm{hr}$ at room temperature, and incubated with primary antibodies diluted in blocking buffer overnight at $4^{\circ} \mathrm{C}$, and secondary antibodies (1:5000 dilution; IR-Dye antibodies, LI-COR) for 1 hour at RT. Membranes were washed in 1X TBS-T and scanned using the Odyssey Infrared Imaging System (LI-COR). Primary antibodies were used as follows: rabbit anti-Gli1 (1:1000; Abcam); goat anti-Gli2 (1:1000; R\&D Systems), rabbit anti-Gli3 (1:100; Santa Cruz), rabbit anti-Pax6 (1:1000 dilution; Cat. \#: 901301, Biolegend); rabbit anti-NeuN (1:1000 dilution; Cat. \#: PA5-784-99, Invitrogen); rabbit anti-GABA A Receptor $\alpha 6$ (1:1000 dilution; Cat. \#. PA5-77403, GABRA6; Invitrogen), and a-Tubulin (1:5000 dilution; Cat. \#: ab4074, Abcam). Quantification and analysis were conducted using the Odyssey Image Studio Software (LI-COR). Protein levels were normalized to GAPDH protein levels. Levels of NeuN and GABRA6 were quantified in correlation with Pax6 levels (NeuN/Pax6 or GABRA6/Pax6) to determine the proportion of Pax6+ cells expressing mature granule neuron markers.

\section{Image Analysis and Acquisition}

Images were acquired using a Nikon E600 microscope equipped with a QCapture Pro camera (QImaging), Zeiss Axioscan Z.1 (Zeiss, Thornwood, NY, USA) using the Zen 2 blue edition software (Zeiss, Thornwood, NY, USA), or the Nikon Ti inverted microscope with CSU-W1 large field of view confocal and Andor Zyla 4.2 sCMOS camera. All images were imported in tiff or jpeg format. Brightness, contrast, and background were adjusted equally for the entire image between controls and mutant using the "Brightness/Contrast" and "Levels" function from "Image/Adjustment" options in Adobe Photoshop or NIH ImageJ without any further modification. NIH Image $J$ was used to threshold background levels between controls and mutant tissues to quantify fluorescence labeling. To quantify cell density, positively labeled cells within defined EGL regions, as defined in Fig. 2A, were counted. Measurements were obtained and averaged from 2-3 $20 \mathrm{~m}$ thick and histologically matched sections encompassing the cerebellar midline (vermis).

\section{Human MB Gene Expression:}

Expression values of FGF5 (ENSG00000138675) were obtained using Geo2R(Barrett et al., 2013) from published human MB subtype expression dataset GEO Accession no. GSE85217 (Cavalli et al., 2017). Analyses were conducted according to reported clinical data, subgroup and subtype classifications accompanying the dataset.

\section{Statistics:}

Prism 8.1 (GraphPad) was used for statistical analysis. Two sample experiments were analyzed by Student's $t$ test and experiments with more than two parameters were analyzed by ANOVA. In 1- or 2- way ANOVA, when interactions were found, follow-up analyses were conducted for the relevant variables using Holm-Sidak's multiple comparisons test. All experiments were conducted at least in triplicate with sample sizes of $\mathrm{n}=$ 3-6 embryos/animals/slices per genotype. $P$ value $\leq 0.05$ was considered statistically significant. Graphs display the mean \pm standard error of the mean (SEM). 
bioRxiv preprint doi: https://doi.org/10.1101/2021.06.23.449636; this version posted June 24, 2021. The copyright holder for this preprint (which was not certified by peer review) is the author/funder, who has granted bioRxiv a license to display the preprint in perpetuity. It is made available under aCC-BY-NC-ND 4.0 International license.

\section{Aberrant FGF signaling in SHH subgroup infantile medulloblastoma}
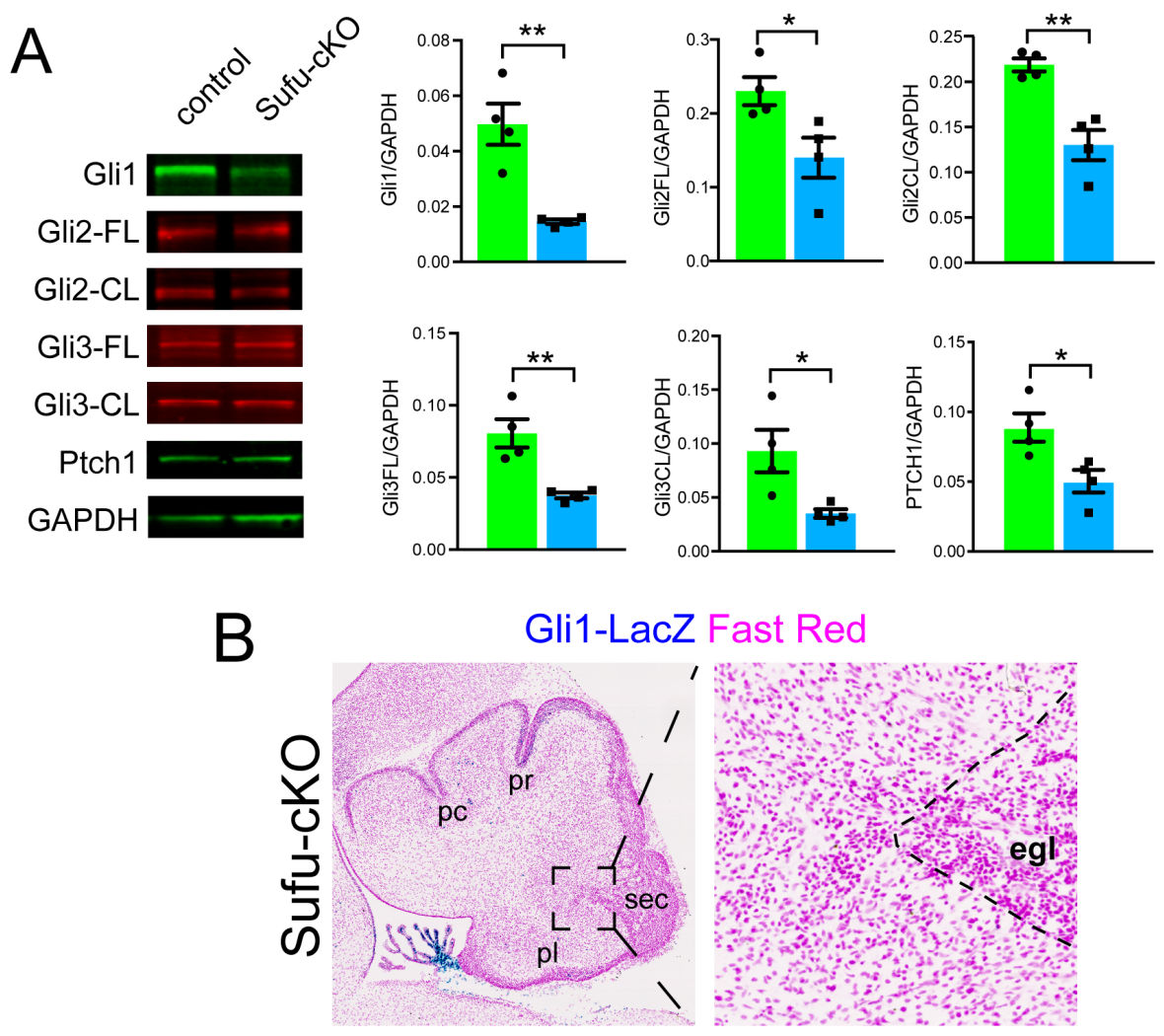

Supplementary Figure 1: Reduced SHH signaling activity in the P0 Sufu-cKO cerebellum. (A) Western blot analysis of cerebellar protein lysates from PO control and Sufu-cKO mice showing significantly lower levels of total and cleaved versions of Gli transcription factors in the PO Sufu-cKO cerebellum. ${ }^{*} \mathrm{p}<0.05,{ }^{* *} \mathrm{p}<0.01$ (B) b-galactosidase activity (blue), representing the Gli1-LacZ transgene, is largely absent in areas adjacent to the EGL along the secondary (sec) fissure of the PO Sufu-cKO cerebellum. 